Methods: A total of 141 patients (103 female, 38 male) who were diagnosed RA according to ACR (American College of Rheumatology) diagnostic criteria were included in the study. Fatigue Symptom Inventory (FSI) was used for evaluation of fatigue. While the disease activity was determined using the Disease Activity Score-28 (DAS28), the Health Assessment Questionnaire (HAQ) was used to determine the functional status. The pain intensity was determined using $10 \mathrm{~cm}$ Visual Analogue Scale-Pain (VAS-pain).

Results: The mean age of the patients is $54.67 \pm 10.70$ years and the mean duration of illness is $14.31 \pm 10.89$ years. When the relationship between fatigue and other factors was examined, a statistically significant relationship was found between FSI fatigue severity scores (maximum, minimum, mean, current), FSI duration scores (number of days felt tired, amount of time felt tired), FSI interference score and $\mathrm{HAQ}$, number of swollen joints, number of tender joints, VAS rest and VAS motion values $(p<0.05)$. There was a statistically significant lower correlation between FSI fatigue severity scores (at least, mean) and DAS28 ( $r: 0.216, r: 0.181$, respectively). There was no significant relationship between FSI scores and age, duration of illness, steroid use

Conclusions: Fatigue affects patients independently of disease duration in patients with RA. Fatigue is associated with disease activity, functional status, and pain. For this reason, fatigue in RA patients should be considered as an important symptom that should not be overlooked and should be struggled.

References:

[1] Steenbergen HW, Tsonaka R, Huizinga TWJ, Boonen A, Helm-van Mill AHM. Fatigue in rheumatoid arthritis; a persistent problem: a large longitudinal study. RMD Open 2015;1:e000041.

[2] Dartel SAA, Repping-Wuts JWJ, Hoogmoed D, Bleijenberg G, Riel PLCM, Fransen J. Association between fatigue and pain in rheumatoid arthritis: does pain precede fatigue or does fatigue precede pain? Arthritis Care Res (Hoboken). 2013 Jun;65(6):862-9.

[3] Singh H, Arya S, Talapatra P, Lather K, Mathur R, Singhania A, Chaudhary V. Assessment of Fatigue in Rheumatoid Arthritis (by Functional Assessment of Chronic IIIness Therapy-Fatigue Score) and Its Relation to Disease Activity and Anemia. J Clin Rheumatol. 2014 Mar;20(2):87-90.

Disclosure of Interest: None declared

DOI: 10.1136/annrheumdis-2017-eular.1689

\section{FRI0145 DOES PAIN HAVE INFLUENCE ON HEALTH ASSESSMENT QUESTIONNAIRE DISABILITY INDEX (HAQ-DI) IN RHEUMATOID ARTHRITIS PATIENT? AN ATTEMPT TO EVALUATE EFFECTIVENESS OF PAIN VAS (PS-VAS) ON HAQ-DI IN REAL CLINICAL PRACTICE -}

I. Yoshii ${ }^{1}$, T. Chijiwa ${ }^{2} .{ }^{1}$ Rheumatology, Yoshii Hospital, Shimanto City;

${ }^{2}$ Rheumatology, Kochi Memorial Hospital, Kochi, Japan

Background: Health Assessment Questionnaire Disability Index (HAQ-DI) is the most important index in treatment for rheumatoid arthritis (RA) patient. HAQ-DI expresses patient's disability in daily life (ADL), and this is influenced by disease activity (ACT-HAQ) and joint structural damage (DAM-HAQ), and aging when patient gets older in senectitude (AGE-HAQ) (1-3). One more factor that possibly makes influence on HAQ-DI is patient's pain. However, this problem is not discussed at all.

Objectives: We have investigate patient's pain and its effect on HAQ-DI in our clinical data in order to evaluate whether pain influences on HAQ-DI, and to make assessment existence of pain related HAQ-DI (PAIN-HAQ)

Methods: RA patients who have been treated continuously for more than five years, who had visited later than October 31th, 2016, were picked up in this study. Patients average 28-joints disease activity score with C-reactive protein (DAS28-CRP), modified HAQ (mHAQ), Sharp/van der Heijde Score (SvdHS), age, and pain score calculated by visual analogue scale (PS-VAS) were calculated in fifth treatment year. Average values of these parameters have been calculated. Relationships among these factors have been investigated statistically using multiple linear regression analysis (MLR). After evaluation of relationship of each pairs of these factors, the relationship between HAQ-DI and the other factors had been evaluated from modified data of these patients in minimize the effect of parameters other than PS-VAS and data that minimized effectiveness of PS-VAS with MLR.

Results: 382 patients had been picked up. Their sex distribution was 87 for male and 295 for female, and their average values and standard deviations of age, DAS28-CRP, HAQ-DI, SvdHS, and PS-VAS were 68.99 and 13.47, 1.91 and $0.54,0.43$ and $0.55,54.97$ and 67.30 , and 22.96 and 17.85 , respectively. HAQ-DI demonstrated significant regression with all of DAS28-CRP, SvdH, age, and PS-VAS $(<0.01)$. DAS28-CRP demonstrated positive correlation with PS-VAS, HAQ-DI, and SvdHS, but negatively correlated significantly with age $(<0.01)$. PS-VAS demonstrated positive correlation with HAQ-DI and DAS28CRP, but negatively correlated with SvdHS significantly $(<0.01)$, while no significant correlation demonstrated with age. SvdHS demonstrated positive correlation with DAS28-CRP and HAQ-DI, but negative correlation with PS-VAS significantly $(<0.01)$, while no significant correlation demonstrated with SvdHS. Age demonstrated positive correlation with HAQ-DI, but negatively correlated with DAS28-CRP $(<0.01)$, while no significant correlation demonstrated with PS-VAS and SvdHS (Figure 1).

After minimizing the data effectiveness of DAS28-CRP, Age, and SvdHS on
HAQ-DI, HAQ-DI demonstrated significant regression only with PS-VAS. When the effectiveness of PS-VAS was minimized, HAQ-DI demonstrated significant regression with parameters other than PS-VAS. Threshold of PS-VAS was $15 \mathrm{~mm}$.

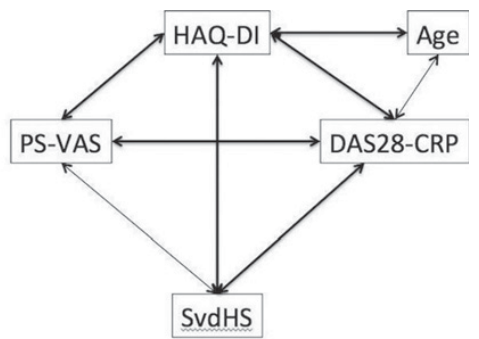

Figure 1: Schematic relationship among HAQ-DI, DAS28-CRP, SvdHS, Age, and PS-VAS. Thick lines demonstrate significant positive correlations, and thin lines demonstrate significant negative correlations

Conclusions: These results suggested that HAQ-DI is influenced PS-VAS when it is no less than $15 \mathrm{~mm}$. Therefore, we conclude that HAQ-DI consists with PAIN-HAQ in adding with ACT-HAQ, DAM-HAQ, and AGE-HAQ.

\section{References:}

[1] Smolen JS, et al. Ann Rheum Dis 2010;69:1058-1064.

[2] Vita AJ, et al. N Engl J Med 1998; 338:1035-1041.

[3] Yoshii I, et al. Arthritis Rheumatol. 2015; 67 (suppl 10).

Disclosure of Interest: None declared

DOI: 10.1136/annrheumdis-2017-eular.3977

\section{FRI0146 ANTI-ELASTIN AND ANTI-ELASTASE AUTOANTIBODIES: POTENTIAL CLINICAL AND DIAGNOSTIC IMPLICATIONS IN RHEUMATOID ARTHRITIS}

N.V. Nenasheva ${ }^{1}$, A.S. Trofimenko ${ }^{1,2}$, I.P. Gontar ${ }^{1}$, L.A. Maslakova ${ }^{1}$

O.A. Rusanova ${ }^{1}$, O.V. Paramonova ${ }^{2} .{ }^{1}$ Research Institute for Clinical and Experimental Rheumatology; ${ }^{2}$ Volgograd State Medical University, Volgograd, Russian Federation

Background: Elastin is an ubiquitous molecule, presented in connective tissue matrix, including skin, ligaments, lungs, and blood vessels. Elastase is a characteristic protease, considerably presented in neutrophils and in pancreas. Autoantibodies $(\mathrm{Ab})$ to elastin and to elastase are promising candidate biomarkers in rheumatoid arthritis (RA).

Objectives: To explore potential clinical and diagnostic utility of anti-elastin and anti-elastase $\mathrm{Ab}$ in RA.

Methods: The research was carried out in agreement with the WMA Declaration of Helsinki principles and was approved by Volgograd Regional Committee on Medical Ethics. All the patients signed the informed consent. We enrolled 106 adult patients with definite RA in Volgograd Municipal Hospital \#25, the diagnosis have been established using ACR-EULAR criteria (2010). For ROC analysis calculations we used mixed control group consisted of 19 patients with ankylosing spondylitis, 32 with gout, 11 with psoriatic arthritis, and 22 with reactive arthritis. Serum anti-elastin and anti-elastase Ab concentrations were evaluated by ELISA, using antigens immobilized on magnetic polyacrylamide beads, which were previously described by our group [1]. Antibody concentrations were expressed as relative optical density units (ODU). The cutoff values for anti-elastin and antielastase $\mathrm{Ab}$ presence were 0.104 and 0.113 ODU, respectively; the calculations were performed using 34 healthy control sera. All the means and operation characteristics were expressed as values (95\% confidence intervals). Differences were considered significant when $\mathrm{p}<0.05$

Results: In RA anti-elastin Ab were found in 37 (34.9\%) patients, and the mean concentration was $0.128(0.118-0.138)$ ODU. There was no significant correlation between DAS28 and anti-elastin concentrations, but the least marker was increased in patients with heart and kidney involvement, as well as in vasculitis patients, comparing to those who have no such manifestations $(p=0.017,0.046$, and 0.009 , respectively). We detected $58(54.72 \%)$ anti-elastase positive RA patients, with the mean concentration $0.137(0.103-0.171)$ ODU. Anti-elastase positive patients had significantly increased frequencies of anemia $(p=0.025)$ and vasculitis $(p=0.017)$ comparing to the negative subgroup. The prevalence of anti-elastase $\mathrm{Ab}$ was also increased along with RA activity. Concentrations of these two $A b$ were positively correlated $(r=0.866, p<0.001)$. For anti-elastin $\mathrm{Ab}$ assay (cutoff point 0.112 ODU) diagnostic sensivity in RA patients was 72 $(62-87) \%$, specificity 50 (41-64)\%, AUC of ROC curve $0.703(0.590-0.797)$. For anti-elastase $\mathrm{Ab}$ assay (cutoff point $0.115 \mathrm{ODU}$ ) the respective values were 77 $(62-87) \%, 81(59-91) \%$, and $0.822(0.675-0.923)$.

Conclusions: Anti-elastin and, increasingly, anti-elastase antibodies are valuable candidate markers to improve diagnosis of RA and, particularly, rheumatoid vasculitis and heart involvement. Further investigations are needed to assess sensivity and specificity of these markers being included in the comprehensive diagnostic algorithms.

References:

[1] Gontar IP, Simakova ES, Trofimenko AS, Zborovskaya IA. An approach for 